\title{
Pertuzumab/Trastuzumab/Docetaxel Followed by FEC Regimen
}

National Cancer Institute

\section{Source}

National Cancer Institute. Pertuzumab/T rastuzumab/Docetaxel Followed by FEC

Regimen. NCl Thesaurus. Code C138044.

A chemotherapy regimen consisting of pertuzumab, trastuzumab and docetaxel, followed by fluorouracil, epirubicin and intravenous cyclophosphamide, used as a neoadjuvant or adjuvant treatment for HER-2/neu-positive breast cancer. 